\title{
High-fidelity direct coherent diffractive imaging of condensed matter
}

\author{
A. J. Morgan, ${ }^{1}$ A. J. D’ Alfonso, ${ }^{1}$ A. V. Martin, ${ }^{2}$ A. I. Bishop,${ }^{3}$ H. M. Quiney, ${ }^{1}$ and L. J. Allen ${ }^{1, *}$ \\ ${ }^{1}$ School of Physics, University of Melbourne, Parkville, Victoria 3010, Australia \\ ${ }^{2}$ Center for Free-Electron Laser Science, DESY, Hamburg 22607, Germany \\ ${ }^{3}$ School of Physics, Monash University, Victoria 3800, Australia
}

(Received 20 June 2011; revised manuscript received 17 August 2011; published 31 October 2011)

\begin{abstract}
We present a deterministic strategy to obtain a high-fidelity reconstruction of the exit-surface wave of a specimen of condensed matter from its diffraction pattern. The direct solution of a set of linear equations extracted from the inverse Fourier transform of the diffraction pattern (which is the autocorrelation of the exit-surface wave) is followed by a simple regularization step in which inconsistencies in the data are corrected. This approach is illustrated using the diffraction pattern of a gnat's wing, illuminated with a laser. We also show that a well-defined residual serves as a proxy for the fidelity of the retrieved exit-surface wave. In addition the residual provides a more stringent test of reconstruction quality than has been previously available in conventional iterative phase retrieval procedures and is easily calculated as an integral component of such methods.
\end{abstract}

DOI: 10.1103/PhysRevB.84.144122

PACS number(s): 42.30.Rx

\section{INTRODUCTION}

The retrieval of the complex exit-surface wave of a specimen of condensed matter from its diffraction pattern using, in particular, coherent sources of $\mathrm{x}$-rays ${ }^{1-7}$ and electrons ${ }^{8-12}$ has recently received a great deal of scientific attention. This approach, known as coherent diffractive imaging (CDI), has the advantage that samples may be imaged without the need to employ post-specimen lens systems or to correct for the aberrations inherent in such optics. The technique has important potential applications in nanoscale science such as, for example, the two- and three-dimensional imaging of viruses $^{13}$ and cells. ${ }^{14}$ It is also an integral component of current efforts to determine the structure of protein molecules using $\mathrm{x}$-ray free-electron laser sources using nanocrystalline or noncrystalline samples ${ }^{15}$ or atomic resolution imaging using electron sources. ${ }^{12}$

The most commonly used techniques for exit-surface wave reconstruction are based on iterative refinement of a trial wave function using the measured diffraction data to provide an amplitude constraint together with additional a priori information about the sample to constrain the phase. These approaches are nonsmooth, nonconvex nonlinear optimization problems, ${ }^{16,17}$ for which a number of outstanding important issues remain. These include difficulties in establishing the uniqueness of the solution under general imaging conditions, the identification of effective convergence criteria, and the associated issue of the choice of a suitable error metric. Uniqueness issues are often addressed in an ad hoc manner in which, for example, the reconstruction procedure is initialized using many different random guesses. The optimal reconstruction may be identified based on a number of criteria: the iteration with the smallest error metric may be selected, the most reproducible reconstruction from random starting guesses may be chosen, or reconstructions may be rejected if the error metric significantly exceeds some threshold. Such criteria possess an unsatisfactorily subjective character, since they do not come with the necessary guarantee that a solution found in such a manner represents a global minimum of the error metric. Furthermore, partial coherence of the illumination, noise, and data missing due to experimental limitations of the imaging configuration may make it difficult or impossible to reconstruct an image successfully using CDI. This combination of procedural and experimental issues renders image reconstruction using iterative CDI methods a challenging task that cannot, in practice, be regarded as a routine procedure.

While iterative CDI methods have certainly been used with great success, parallel efforts have continued to develop practical, noniterative methods that determine solutions directly. These efforts have largely been directed toward addressing outstanding issues regarding the uniqueness of reconstructions and to the characterization of the effects of experimental errors on reconstruction quality. Several of these approaches are based on holography, in which the interference is measured between a known wave, involving either the incident illumination itself or a well-characterized reference wave, and the unknown scattered wave from the object. ${ }^{18-22}$ A recent variation on this idea utilizes the interference generated by the wave that scatters from the target object and another wave that scatters from the edges of a rectangular aperture located in the plane of the target object. ${ }^{23}$ The principal aim of this approach was the construction of an analytic solution to the imaging problem using the well-known properties of waves scattered from such apertures in conjunction with a restriction imposed on the positioning of the target object within the aperture. It has subsequently been applied to experimental data obtained using visible light. ${ }^{24}$

The direct method proposed in Ref. 25 and developed further here does away with the restrictive imaging requirements specified in Ref. 23. The approach possesses the advantage that it is general, offering great flexibility in the choice of the reference wave that forms part of the solution procedure and facilitating its application to a wide variety of experimental conditions. It is, furthermore, applicable when the reference is simply the unscattered part of the illuminating beam. No assumptions are made about the strength of the scattering by the specimen, so that it is applicable even if strong phase and amplitude variations are present in the scattered wave. In a preliminary, proof-of-principle demonstration of this method using optical data, ${ }^{26}$ the diffraction pattern was cropped to lessen the computational burden. In 
addition, the adverse effects of noise were ameliorated by the introduction of a method to precondition the intensity information. This was sufficient to produce a clean image but at the expense of resolution and increased computational time.

In this article we demonstrate that high-resolution experimental diffraction data may readily be incorporated within this scheme by the introduction of a simple regularization procedure. This approach provides a much more robust way to stabilize the method of Ref. 25 with respect to noise without resolution loss or the additional computational expense of preconditioning. These new developments give a greatly improved reconstruction of the gnat's wing to that in Ref. 26, achieving a level of detail comparable to the microscope image. This represents a significant step in making the method a viable approach when applied to more challenging experimental situations, such as those encountered in nanoscale imaging using $\mathrm{x}$-rays or electrons. As an adjunct to this, an analysis of the robustness of this noniterative approach to the effects of noise has also enabled us to formulate a new scheme to monitor the performance of conventional, iterative CDI techniques. It is also shown that one may readily construct an error metric that indicates the quality of the reconstructed exit-surface wave from the target object in a least-squares sense. This may be contrasted with conventional error metrics in iterative CDI, which measure how well the diffraction data are reproduced in a simulation of the scattering experiment. The comparative use of these error metrics provides insights into the relative performances of the iterative and noniterative procedures and offers a new quantitative measure of the quality of any reconstruction from coherent diffraction data.

\section{THEORETICAL BACKGROUND}

As discussed in Ref. 25, the formulation of the CDI phase problem as the solution of a linear system begins with the assumption that the coherent complex wave function $\psi_{\mathrm{e}}$ in the exit-surface plane of the object is decomposed as

$$
\psi_{\mathrm{e}}=\psi_{\mathrm{illum}}+\psi_{\mathrm{obj}}
$$

where $\psi_{\text {illum }}$ is the wave function representing the known incident illumination (and any known reference scatterers) while $\psi_{\text {obj }}$ is the unknown scattered wave from the object. This decomposition is purely a matter of convenience, separating the known wave $\psi_{\text {illum }}$ from the unknown wave $\psi_{\text {obj }}$ and does not contain any approximation about the strength of the scattering. The linear formulation of the CDI phase problem is possible when the interference, after propagation to the detector plane, between the known $\psi_{\text {illum }}$ and the unknown $\psi_{\text {obj }}$, can be extracted from the diffraction intensities. This interference signal is related to the cross-correlation between these functions, which in turn can form the basis of a set of linear equations. A linearly independent or overdetermined set of linear equations can be obtained provided the imaging system meets well-defined geometric conditions.

The conditions that must be met by the imaging system are detailed in Ref. 25 and are recalled briefly here. In practice, these conditions are readily satisfied under experimental imaging conditions. We require that the object must be of
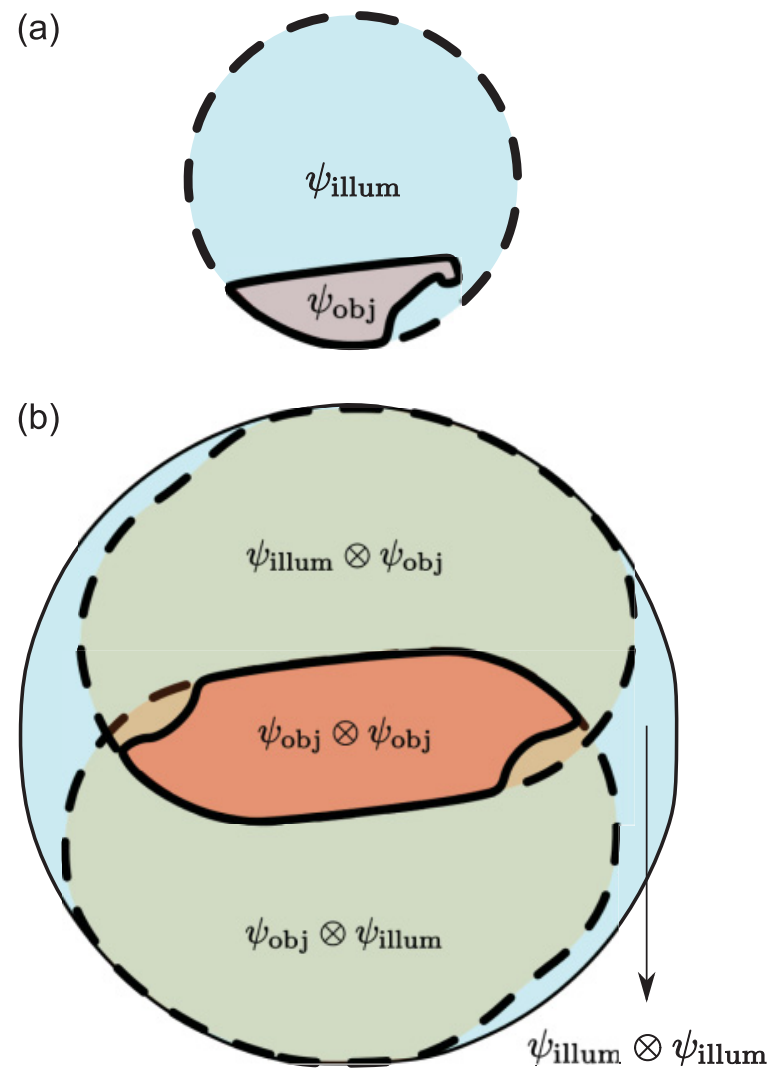

FIG. 1. (Color online) A schematic diagram of the geometric regions discussed in Sec. II. (a) The exit-surface plane of the object indicating the area occupied by the object (solid outline) within the circular uniform illumination (dashed outline). (b) The corresponding schematic of the autocorrelation plane. The autocorrelation of the finite object is confined to a finite region (marked by the thick solid line). The dashed lines outline the region containing the cross-correlation between the sample and the illumination, which overlaps the autocorrelation of the object. The thin solid line marks the region that contains the autocorrelation of the illumination. The linear equations in Eq. (4) are set up by subtracting the known autocorrelation of the illumination and considering only the area containing cross-terms which is outside the autocorrelation of the object.

finite extent, or that only a finite portion of the object is illuminated. The illumination must be at least twice as large as the illuminated part of the object, and it must contain some nonuniformity in its intensity or phase. This variation must be at least one object diameter away from the object, a condition that is readily satisfied under a wide variety of experimental conditions. A suitable variation in the illumination could be, for example, the edge of the circular beam or the asymptotic profile of a Gaussian beam. An example of such an exit wave is shown in Fig. 1(a), where the solid line marks the finite region of a sample (the solid line) that is illuminated by a pencil probe (the region marked by the dashed lines), which varies in intensity one diameter away from the object region due to the circular edge. In the specific case in which the reference scatterer is a pinhole, this method corresponds to Fourier holography. ${ }^{25}$ 
The inverse Fourier transform of the experimentally measured diffraction pattern $I_{\text {expt }}$ yields the autocorrelation of the exit-surface wave:

$$
f \equiv \mathcal{F}^{-1}\left[I_{\text {expt }}\right]=\psi_{\mathrm{e}} \otimes \psi_{\mathrm{e}},
$$

where, for example, $a \otimes b=\mathcal{F}^{-1}\left[A^{*} B\right], A=\mathcal{F}[a], \quad B=$ $\mathcal{F}[b]$, and $\mathcal{F}$ denotes the Fourier transform operator. Consequently, using Eq. (1), we can write

$$
\begin{aligned}
f= & \psi_{\text {illum }} \otimes \psi_{\text {illum }}+\psi_{\text {illum }} \otimes \psi_{\text {obj }} \\
& +\psi_{\text {obj }} \otimes \psi_{\text {illum }}+\psi_{\text {obj }} \otimes \psi_{\text {obj }}
\end{aligned}
$$

Our aim is to separate the cross-correlations, $\psi_{\text {illum }} \otimes \psi_{\text {obj }}$ and $\psi_{\text {obj }} \otimes \psi_{\text {illum }}$, which can be solved as a linear system for $\psi_{\mathrm{obj}}$, from the nonlinear autocorrelation $\psi_{\mathrm{obj}} \otimes \psi_{\mathrm{obj}}$. The geometric conditions, which are described above, indicate when this separation is possible. To see how the spatial separation of these correlations works, consider the example shown in Fig. 1. Figure 1(b) illustrates the domain of the four correlation terms in Eq. (3) using the representations of $\psi_{\mathrm{obj}}$ and $\psi_{\text {illum }}$ in Fig. 1(a). The autocorrelation of the object, $\psi_{\mathrm{obj}} \otimes \psi_{\mathrm{obj}}$, is confined to the region delineated by the thick solid line. The terms expressing the cross-correlation between the sample and the illumination, $\psi_{\text {illum }} \otimes \psi_{\text {obj }}$ and $\psi_{\text {obj }} \otimes \psi_{\text {illum }}$, which are linear in $\psi_{\mathrm{obj}}$, are confined to the areas bounded by the thick dashed lines. Last, the autocorrelation of the illumination, $\psi_{\text {illum }} \otimes \psi_{\text {illum }}$, covers the circular region enclosed by the thin solid line. Since $\psi_{\text {illum }}$ is assumed to be well characterized, $\psi_{\text {illum }} \otimes \psi_{\text {illum }}$ can be calculated and subtracted from $f$. Hence, the remaining function $f-\psi_{\text {illum }} \otimes \psi_{\text {illum }}$ can be partitioned geometrically into regions that depend linearly and nonlinearly on $\psi_{\text {obj. }}$. The linear regions are those where $\psi_{\text {obj }} \otimes \psi_{\text {obj }}=0$. It is important to note that after the $\psi_{\text {illum }} \otimes \psi_{\text {illum [marked }}$ by the thin solid line in Fig. 1(b)] is subtracted from the autocorrelation function of the exit wave in Eq. (3) not all of the cross-correlation terms are available or needed. Indeed, those contributions where $\psi_{\mathrm{obj}} \otimes \psi_{\mathrm{obj}} \neq 0$ have been excluded in the present case. These linear equations, which in general form an overdetermined set, can be expressed in the form

$$
\mathbf{A x}=\mathbf{b} .
$$

The elements of the matrix $\mathbf{A}$ are determined by the values of the known wave $\psi_{\text {illum }}$, the vector $\mathbf{x}$ contains the values of the unknown wave, $\psi_{\mathrm{obj}}$, and $\mathbf{b}$ contains values of $\psi_{\text {illum }} \otimes \psi_{\mathrm{obj}}+$ $\psi_{\text {obj }} \otimes \psi_{\text {illum }}$ drawn from the the region where $\psi_{\text {obj }} \otimes \psi_{\text {obj }}$ vanishes.

Equation (4) can be solved for the unknown object $\psi_{\text {obj }}$ by standard least-squares techniques. Solutions obtained in this manner represent least-squares approximations of $\mathbf{x}$, which, in this case, represents the available scattering information about the object. In contrast, conventional CDI based on iterative solution of nonlinear equations constructs leastsquares approximations to the measured diffraction pattern.

\section{ANALYSIS OF EXPERIMENTAL DATA}

As an illustration of a direct, high-resolution, regularized CDI retrieval, as discussed above, we use the diffraction pattern formed by an Australian gnat's wing using a $\mathrm{HeNe}$ laser (wavelength $632.8 \mathrm{~nm}$ ) and shown in Fig. 2(a). The

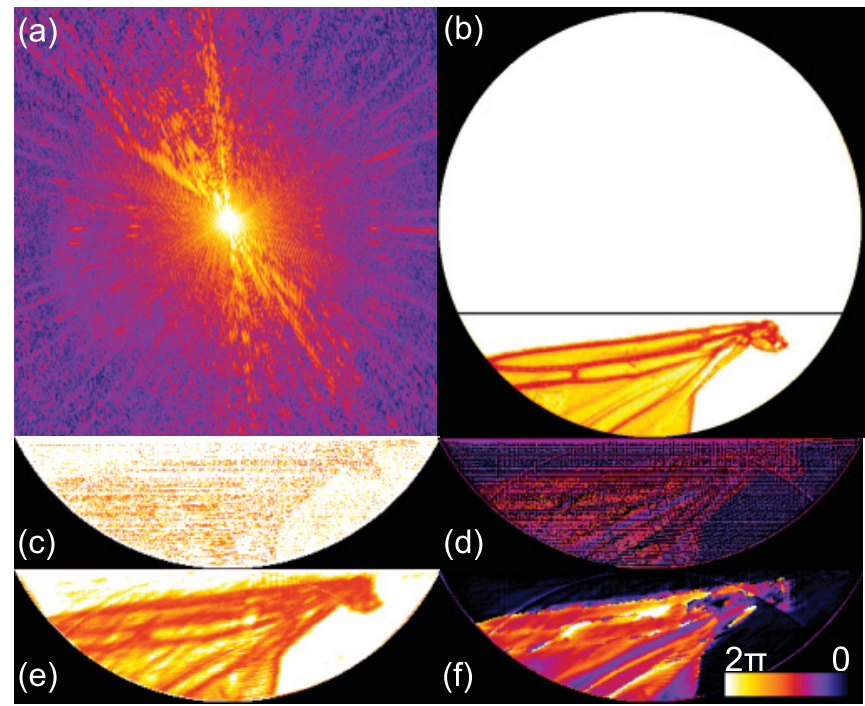

FIG. 2. (Color online) (a) The far-field diffraction pattern of a gnat's wing illuminated with a HeNe laser. (b) A high-resolution microscope image of the gnat's wing and illuminating beam. The black horizontal line indicates the area assumed to contain the illuminated portion of the object in the linear retrieval method, which yields in (d) the retrieved intensity and in (c) the change in phase of the incident wave due to the wing. The regularized image is shown in (e) and the phase in (f).

incident beam was attenuated using a neutral density filter before spatial filtering by focusing through a $20 \mu \mathrm{m}$ pinhole. It was then recollimated by a lens of focal length $250 \mathrm{~mm}$ to obtain an approximately planar monochromatic beam. This beam then illuminated a conical hole formed in a $3 \mathrm{~mm}$ thick stainless-steel disk with the sides of the hole tapered by $15^{\circ}$ and with the larger diameter facing the beam. The object was fixed with adhesive to the outside of the smaller-diameter aperture, which had a diameter of $2.035 \pm 0.005 \mathrm{~mm}$. The tapering of the hole avoided diffraction effects associated with the first aperture. An antireflection coated, planoconvex lens of focal length $75 \mathrm{~mm}$ was located $1 \mathrm{~mm}$ downstream of the object and focused onto the surface of a monochrome CCD camera (a Prosilica GE1650). This CCD has $1600 \times 1200$ pixels that are each $7.4 \mu \mathrm{m}$ squared and covers a 12-bit per pixel dynamic range, ensuring that $\psi_{\text {illum }}+\psi_{\text {obj }}$ was sampled to high resolution in the detector plane

So in this case, $\psi_{\text {illum }}$ represents a near-uniform illumination confined to the circular aperture shown in Fig. 2(b), while $\psi_{\text {obj }}$ represents the modification to $\psi_{\text {illum }}$ caused by its transit through the wing, which is assumed to be confined to the small region bounded by the black semicircle, which outlines the edge of the aperture and the horizontal line in Fig. 2(b). Unlike in the schematic in Fig. 1(a), where we tacitly assumed that we knew precisely the region covered by the object, we are free to assume a region that we believe contains the object. Provided this region still satisfies the conditions required for a solution, as outlined in Ref. 25, everything goes through as discussed in Sec. II but with the area occupied by $\psi_{\mathrm{obj}} \otimes \psi_{\mathrm{obj}}$ now defined by the area assumed to contain the object. In other words, the reconstruction is performed over a region that is larger in spatial extent than the scattering object. This 
augmented, "effective object" incorporates a region containing only the illumination. This introduces a compromise in which the number of equations is decreased while simultaneously increasing the number of unknowns to be solved. Unlike the commonly used iterative techniques, which are known to be sensitive to the sample support, our procedure is not critically dependent on a detailed knowledge of the physical support of the scattering object.

The results of the initial linear step in the reconstruction are shown in Fig. 2(c), where the image is displayed, and in Fig. 2(d), where the modification of the phase of the incident illumination by the wing is shown. The reconstruction used data from a much wider scattering angle than in Ref. 26. The present study demanded the use of two additional computational techniques: (1) the use of bicubic interpolation to apply a subpixel shift to the diffraction pattern to ensure optimal alignment with the optical axis of the imaging system and (2) significant additional computational resources. The reconstruction involved 311632 equations and 51026 unknown amplitudes of $\psi_{\mathrm{obj}}$. The diameter and normalization of the illumination relative to the diffraction pattern (or, equivalently, the level of absorption in the sample) were determined by identifying the portion of the autocorrelation function that depended only on the illumination [see the schematic in Fig. 1(b)]. The retrieval was insensitive to renormalization of the diffraction pattern relative to the illumination in the range $0.97 \pm 0.048$. These results took approximately 12 hours of CPU time on a 12 core AMD Opertron $2.0 \mathrm{GHz}$ computer. Solution of the equations using a single-precision implementation of the QR decomposition required $70 \mathrm{~GB}$ of RAM. Although this is a longer computing time than a single standard iterative CDI reconstruction, which takes considerably less than an hour, it is much less total time than that required for tens or hundreds of reconstructions typically performed to find suitable parameters and verify the solution of a standard iterative method. The direct nature of the method potentially saves the researcher many hours of exploratory investigation. The destabilizing effects of finite word-length arithmetic on the determination of $\mathbf{x}$ using Eq. (4) depends on the reciprocal of the condition number of the coefficient matrix, A. In the current case this quantity is $7.0 \times 10^{-6}$, indicating that single-precision arithmetic is sufficient to determine $\mathbf{x}$.

In the direct solution of Eq. (4), shown in Fig. 2(c) and Fig 2(d), the structure of the wing is obscured by the effects of measurement noise, stray light scatter, misalignment of $\psi_{e}$ and $\psi_{\text {illum }}$, and by reconstruction artifacts, such as the phantom reconstruction of an edge of $\psi_{\text {illum. The reconstruction }}$ quality of $\psi_{\text {obj }}$ may be dramatically improved by a simple regularization procedure, producing Fig. 2(e) and Fig 2(f), which compare much more favorably to the microscope image, Fig. 2(b), than the results in Ref. 26, where the data were truncated in the diffraction plane and a preconditioning step requiring additional computational resources was applied. The substantial improvement seen here allows the wing structure to be interpreted, and the phase even shows variations due to the orientation of the wing membrane, which are not accessible in the microscope image.

Before detailing the regularization procedure, we will first explain how the measurement errors arise. Implicit in the reconstruction procedure is a knowledge of $\psi_{\text {illum, the far- }}$ field diffraction data involving both the illumination, $I_{\text {illum }}=$ $\left|\mathcal{F}\left[\psi_{\text {illum }}\right]\right|^{2}$, and the illumination perturbed by the scattering object, $I_{\mathrm{e}}=\left|\mathcal{F}\left[\psi_{\mathrm{e}}\right]\right|^{2}$. The construction of the linear equations that define Eq. (4) may be written in the symbolic form

$$
\begin{aligned}
& {\left[\psi_{\text {illum }} \otimes \psi_{\text {obj }}+\psi_{\text {obj }} \otimes \psi_{\text {illum }}\right]_{\mathbf{x} \in \mathcal{D}}} \\
& =\left[\mathcal{F}^{-1}\left[I_{\mathrm{e}}-I_{\text {illum }}\right]\right]_{\mathbf{x} \in \mathcal{D}},
\end{aligned}
$$

where, as discussed, the exclusion of the terms that depend on $\psi_{\text {obj }} \otimes \psi_{\text {obj }}$ in $I_{\mathrm{e}}-I_{\text {illum }}$ is achieved by the judicious specification of a domain $\mathcal{D}$ on $\mathbf{x}$.

In the ideal case in which both $I_{\mathrm{e}}$ and $I_{\text {illum }}$ are free from measurement errors, we expect that the solution of Eq. (4) determines $\psi_{\text {obj }}$ with the full precision allowed by finite word-length arithmetic; in simulation studies this expectation is realized. ${ }^{25}$ The evidence presented in Fig. 2(c) and Fig. 2(d) indicates, however, that measurement errors in experimental data may have a strong influence on the quality with which $\psi_{\text {obj }}$ is determined. On the assumption that $\psi_{\text {illum }}$ is well characterized, the linear character of Eq. (5) enables one to write

$$
I_{\text {expt }}-I_{\text {illum }}=I_{\mathrm{e}}+I_{\delta}-I_{\text {illum }},
$$

in which $I_{\text {expt }}$ is the experimental measurement of the quantity assumed to be $I_{\mathrm{e}}$ in Eq. (5) and $I_{\delta}$ serves to collect together all deviations of $I_{\text {expt }}$ from $I_{\mathrm{e}}$ and the ideal formulation represented by Eq. (5) due to all sources. The domain specification in Eq. (5) has the effect of simply redefining $\psi_{\text {obj }}$ to include both the target function and a correction factor, so that $\psi_{\text {obj }}^{\prime}=\psi_{\text {obj }}+\psi_{\delta}$. The factor $\psi_{\delta}$ represents a phantom object that provides the best least-squares compensation for $I_{\delta}$, subject to the same domain restriction, $\mathcal{D}$, as $\psi_{\text {obj }}$.

The adverse effects of $\psi_{\delta}$ on the reconstruction quality of $\psi_{\text {obj }}$ can be dramatically reduced by a simple regularization procedure. The wave $\psi_{\mathrm{obj}}^{\prime}$ is assembled from the elements of $\mathbf{x}$ and propagated to the detector plane, where it is added to $\psi_{\text {illum }}$. The amplitude of $\psi_{\text {illum }}+\psi_{\text {obj }}^{\prime}$ is then rescaled to reproduce the measured diffraction intensities. The illumination, $\psi_{\text {illum }}$ is next subtracted from the rescaled complex wave field, and the resulting representation of $\psi_{\mathrm{obj}}^{\prime}$ is propagated back to the exit surface of the scattering object. This regularization procedure may be written as the projection $\mathcal{P}_{\text {mod }} \psi_{\text {obj }}^{\prime} \mapsto \psi_{\text {obj }}^{\prime}$ and is identical to the application of the Fourier modulus constraint in iterative phase retrieval methods such as the error-reduction procedure (ER) or the hybrid input-output procedure (HIO).

A single application of $\mathcal{P}_{\text {mod }}$ to the direct solution of Eq. (4) results in a dramatic improvement in the quality of the reconstruction, the amplitude and phase of which are displayed, respectively, in Figs. 2(e) and 2(f). The internal structure of the wing is now clearly evident, and reconstruction artifacts are no longer prominent. In the present case, we have found that the most significant contribution to $\psi_{\delta}$ comes from a slight displacement of $\psi_{\text {expt }}$ relative to $\psi_{\text {illum }}$, which causes a residual signal to propagate from the edge of the illumination. Since the solution procedure rigorously imposes the previously defined support on the reconstruction of $\psi_{\mathrm{obj}}$, the effect is to absorb signal due to the misalignment in phaseshifted segments within the reconstruction. The regularization procedure may be used to separate $\psi_{\mathrm{obj}}$ and $\psi_{\delta}$ from $\psi_{\mathrm{obj}}^{\prime}$. The amplitude of $\psi_{\text {obj }}$ and $\psi_{\delta}$ are shown in Figs. 3(a) and 3(b), respectively. The effect of the regularization procedure is to 


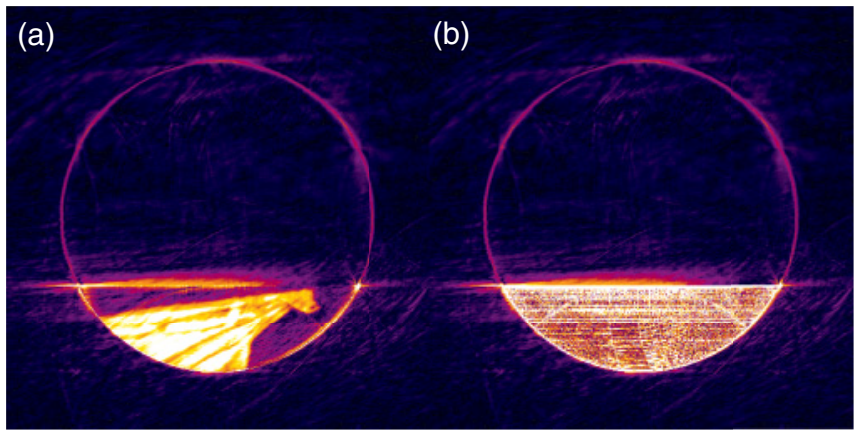

FIG. 3. (Color online) Amplitude of (a) $\psi_{\text {obj }}$ and (b) $\psi_{\delta}$.

propagate the information within the support in Fig. 3(b) onto the bright halo that appears in Fig. 3(a). The application of the regularization procedure facilitates the identification and removal of these spurious contributions because they mostly lie outside the support after the application of $\mathcal{P}_{\text {mod }}$. Any misalignment of the illumination has little influence on the quality of the reconstruction inside the support.

\section{ERROR METRICS}

A quantitative measure of reconstruction quality can be obtained by calculating the residual, $\varepsilon_{\text {residual }}$, defined by

$$
\varepsilon_{\text {residual }}=\frac{\left(\mathbf{x}-\mathbf{x}_{0}\right)^{T} \mathbf{A}^{T} \mathbf{A}\left(\mathbf{x}-\mathbf{x}_{0}\right)}{\mathbf{x}_{0}^{T} \mathbf{A}^{T} \mathbf{A} \mathbf{x}_{0}},
$$

where $\mathbf{x}$ is the retrieved image and $\mathbf{x}_{0}$ is the unknown, target reconstruction. In this form, $\mathbf{x}_{0}$ appears only in the forms $\mathbf{A} \mathbf{x}_{0}=\mathbf{b}$ or $\mathbf{x}_{0}^{T} \mathbf{A}^{T}=\mathbf{b}^{T}$, which are known quantities derived from experimental data. The evaluation of $\varepsilon_{\text {residual }}$ proves to be dominated by contributions involving the diagonal elements of $\mathbf{A}^{T} \mathbf{A}$, so it is well approximated by

$$
\varepsilon_{\text {residual }} \simeq\left\langle\operatorname{Tr} \mathbf{A}^{T} \mathbf{A}\right\rangle \frac{\left(\mathbf{x}-\mathbf{x}_{0}\right)^{T}\left(\mathbf{x}-\mathbf{x}_{0}\right)}{\mathbf{x}_{0}^{T} \mathbf{A}^{T} \mathbf{A} \mathbf{x}_{0}} .
$$

The summation over all diagonal contributions from $\mathbf{A}^{T} \mathbf{A}$ can be approximated by an average over its trace, $\left\langle\operatorname{Tr} \mathbf{A}^{T} \mathbf{A}\right\rangle$. All other contributions involving the off-diagonal elements of $\mathbf{A}^{T} \mathbf{A}$ are suppressed by the rapid fluctuation of the elements of $\mathbf{x}-\mathbf{x}_{0}$ about zero, the mean value in a least-squares fitting procedure. Apart from an overall scale factor, therefore, we expect $\varepsilon_{\text {residual }}$ to possess similar behavior to the fidelity, $\varepsilon_{\text {fidelity }}$,

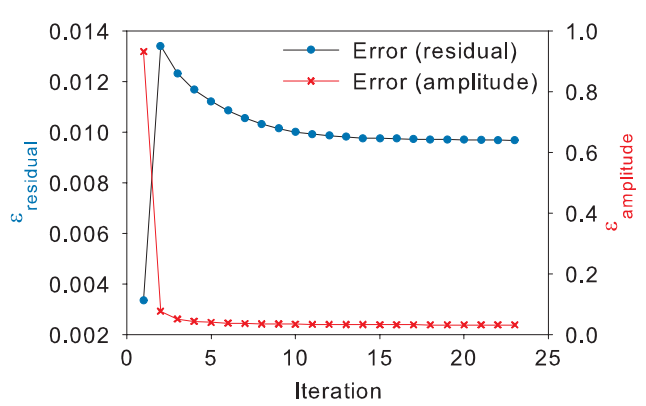

FIG. 4. (Color online) Comparison of $\varepsilon_{\text {residual }}$ and $\varepsilon_{\text {amplitude }}$ as a function of iteration number, for 23 iterations of the ER procedure using the solution of Eq. (5) as the seed.

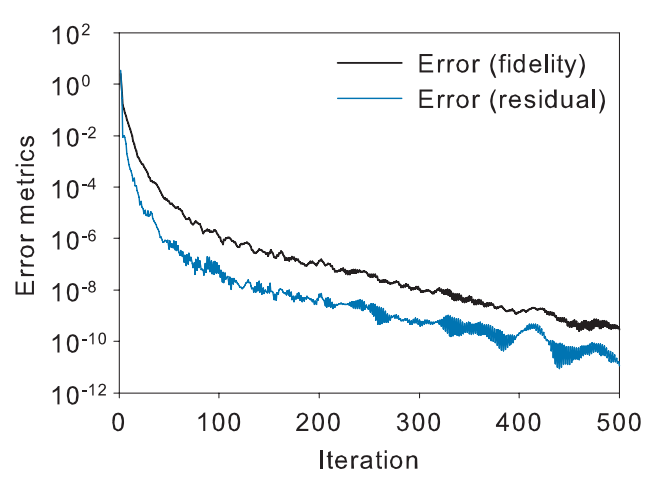

FIG. 5. (Color online) Comparison of $\varepsilon_{\text {fidelity }}$ and $\varepsilon_{\text {residual }}$ as a function of iteration number for 500 iterations of the HIO procedure for a model problem seeded with a random guess.

defined by

$$
\varepsilon_{\text {fidelity }}=\frac{\left(\mathbf{x}-\mathbf{x}_{0}\right)^{T}\left(\mathbf{x}-\mathbf{x}_{0}\right)}{\mathbf{x}_{0}^{T} \mathbf{x}_{0}} .
$$

In order to demonstrate the complementary character of the least-squares fitting procedure implicit in the solution of Eq. (5) and the steepest-descent nature of the conventional error-reduction procedure we have monitored $\varepsilon_{\text {residual }}$ and $\varepsilon_{\text {amplitude }}$ in an iterative refinement of $\psi_{\text {obj }}$, which is shown in Fig. 4. The metric $\varepsilon_{\text {amplitude }}$ is

$$
\varepsilon_{\text {amplitude }}=\frac{\sum_{n}\left(\sqrt{I}_{\text {expt }}-\left|\Psi_{\mathrm{e}}\right|\right)^{2}}{\sum_{n} I_{\text {expt }}}
$$

and is widely employed in assessing the quality of images obtained by CDI. ${ }^{16}$ It is clear that on the first application of $\mathcal{P}_{\text {mod }}, \varepsilon_{\text {residual }}$ rises sharply, because the new solution is no longer the best least-squares fit to the object. Conversely, $\varepsilon_{\text {amplitude }}$ falls rapidly, and the behavior in both cases is due to a violation of the support constraint in the initial guess for $\psi_{\mathrm{obj}}$.

Given that Eq. (7) is easily computed using FFTs, this suggests its use as an error metric in conventional nonlinear iterative phase retrieval methods and as a proxy to the otherwise inaccessible $\varepsilon_{\text {fidelity }}$. In order to test the use of Eq. (7) as an error metric in a conventional nonlinear iterative phase retrieval, a simulation of the gnat's wing was devised, based on Fig. 2(b). A conventional HIO procedure was used, and there was no misalignment of the illumination and the diffraction data. This procedure was run for 500 iterations, and both $\varepsilon_{\text {fidelity }}$ and $\varepsilon_{\text {residual }}$ calculated at each iteration.

Examination of Fig. 5 reveals that $\varepsilon_{\text {residual }}$ closely tracks $\varepsilon_{\text {fidelity }}$ to within a constant after the first few iterations, reproducing almost all of its dominant qualitative features. This suggests that the residual, which is accessible if the linear imaging conditions are met, is a reliable error metric for conventional iterative phase retrieval.

\section{CONCLUSIONS}

We have shown that it is possible to find an exit-surface wave of high fidelity from experimental diffraction data by a direct procedure in which the linear information is identified within the autocorrelation of an exit-surface wave under given illumination conditions, Eq. (3). The approach leads to 
an overdetermined system of linear equations, Eq. (4), the solution of which is refined subsequently by a single-step regularization procedure. This direct approach eliminates the highly chaotic character of the parameter search in iterative procedures and can be thought of as providing a starting point to a very rapidly convergent iterative approach (one iteration, the regularization step, suffices). The regularization step was shown to significantly reduce the effects of noise and experimental error, outperforming the approach of preconditioning Eq. (4). This result raises expectations that the direct method combined with refinement is ready to deliver similar results in $\mathrm{x}$-ray and electron applications, where the scientific outcomes are potentially of greater interest.
Finally, it has also been shown that the residual, $\varepsilon_{\text {residual }}$, provides a quantitative measurement of the quality of the reconstruction if one chooses an iterative phase retrieval method at the outset, such as the ER or HIO procedures. This metric is as reliable and informative as $\varepsilon_{\text {fidelity }}$ and assumes only that the linear imaging conditions are satisfied and that the residual can be calculated.

\section{ACKNOWLEDGMENTS}

HMQ and LJA acknowledge support from the Australian Research Council. *1ja@unimelb.edu.au

${ }^{1}$ H. N. Chapman et al., Nature (London) 470, 73 (2011).

${ }^{2}$ H. M. Quiney, J. Mod. Opt. 57, 1109 (2010).

${ }^{3}$ K. A. Nugent, Adv. Phys. 59, 1 (2010).

${ }^{4}$ B. Abbey, G. J. Williams, M. A. Pfeifer, J. N. Clark, C. T. Putkunz, A. Torrance, I. McNulty, T. M. Levin, A. G. Peele, and K. A. Nugent, Appl. Phys. Lett. 93, 214101 (2008).

${ }^{5}$ A. Schropp et al., Appl. Phys. Lett. 96, 091102 (2010).

${ }^{6}$ D. R. Solli, S. Gupta, and B. Jalali, Appl. Phys. Lett. 95, 231108 (2009).

${ }^{7}$ Z. Wang and G. Popescu, Appl. Phys. Lett. 96, 051117 (2010).

${ }^{8}$ C. Dwyer, A. I. Kirkland, P. Hartel, H. Müller, and M. Haider, Appl. Phys. Lett. 90, 151104 (2007).

${ }^{9}$ O. Kamimura, K. Kawahara, T. Doi, T. Dobashi, T. Abe, and K. Gohara, Appl. Phys. Lett. 92, 024106 (2008).

${ }^{10}$ R. Dronyak, K. S. Liang, Y. P. Stetsko, T.-K. Lee, C.-K. Feng, J.-S. Tsai, and F.-R. Chen, Appl. Phys. Lett. 95, 111908 (2009).

${ }^{11}$ R. Dronyak, K. S. Liang, J.-S. Tsai, Y. P. Stetsko, T.-K. Lee, and F.-R. Chen, Appl. Phys. Lett. 96, 221907 (2010).
${ }^{12}$ L. De Caro, E. Carlino, G. Caputo, P. D. Cozzoli, and C. Giannini, Nature Nanotechnol. 5, 360 (2010).

${ }^{13}$ M. M. Seibert et al., Nature (London) 470, 79 (2011).

${ }^{14}$ D. Shapiro et al., Proc. Natl. Acad. Sci. USA 102, 15343 (2005).

${ }^{15}$ R. Neutze, R. Wouts, D. van der Spoel, E. Weckert, and J. Hajdu, Nature (London) 406, 752 (2000).

${ }^{16}$ J. R. Fienup, Appl. Opt. 21, 2758 (1982).

${ }^{17}$ S. Marchesini, Rev. Sci. Instrum. 78, 011301 (2007).

${ }^{18}$ S. Eisebitt, J. Luning, W. F. Schlotter, M. Lorgen, O. Hellwig, W. Eberhardt, and J. Stohr, Nature (London) 432, 885 (2004).

${ }^{19}$ H. Lichte and M. Lehmann, Rep. Prog. Phys. 71, 016102 (2008).

${ }^{20}$ S. Marchesini et al., Nature Photonics 2, 560 (2008).

${ }^{21}$ M. Guizar-Sicairos and J. R. Fienup, Opt. Lett. 33, 2668 (2008).

${ }^{22}$ D. Zhu et al., Phys. Rev. Lett. 105, 043901 (2010).

${ }^{23}$ S. G. Podorov, K. M. Pavlov, and D. M. Paganin, Opt. Express 6, 9954 (2007).

${ }^{24}$ S. G. Podorov, A. I. Biship, D. M. Paganin, and K. M. Pavlov, Ultramicroscopy 111, 782 (2011).

${ }^{25}$ A. V. Martin and L. J. Allen, Opt. Commun. 281, 5114 (2008).

${ }^{26}$ A. V. Martin, A. I. Bishop, D. M. Paganin, and L. J. Allen, Ultramicroscopy 111, 777 (2011). 